\title{
Comportamiento cíclico drenado y diseño de revancha mínima en tranques de relaves
}

Drained cyclic behaviour and minimum freeboard design in tailings dams

Fecha de entrega: 10 de diciembre 2018 Fecha de aceptación: 24 de abril 2019

\section{Cristian Monje y Gonzalo Suazo}

Departamento de Ingeniería Civil, Universidad Técnica Federico Santa María, Avenida España 1680, Valparaíso, Chile, cristian.monje.robles@gmail.com,gonzalo.suazo@usm.cl

La fracción gruesa de los relaves (arenas) se utiliza para construir el muro principal en tranques de relaves. Debido a la importancia de esta estructura en la estabilidad global del depósito, está diseñada para resistir diferentes tipos de carga, como por ejemplo cargas estáticas, por infiltración, dinámicas, eólicas, entre otras. En este sentido, una de las mayores preocupaciones de los ingenieros geotécnicos es la respuesta no drenada de las arenas ante la eventual ocurrencia de fenómenos de licuefacción. Por otra parte, la respuesta cíclica drenada de arenas de relaves recibe poca atención a pesar de sus posibles implicaciones en la ocurrencia de fenómenos como el overtopping. Lo anterior, se podría deber a que en la práctica existen recomendaciones generales que especifican la revancha operacional y total mínima de un tranque de relaves. Pese a lo anterior, es incierto si estas especificaciones son adecuadas para un amplio rango de materiales y condiciones sísmicas a las que pudiese estar sometida la estructura. En este contexto, el presente estudio muestra los resultados de pruebas DSS (Direct Simple Shear) drenadas realizadas en relaves de arena preparados a diferentes densidades y bajo diversas solicitaciones sísmicas. Los resultados se presentan en términos de asentamiento versus esfuerzo cíclico aplicado. Además, se realizaron pruebas monótonas para explorar de forma preliminar la relación entre la línea de estado crítico y la respuesta cíclica observada. Esto permitió encontrar una relación entre el asentamiento, la demanda cíclica y el parámetro de estado crítico.

Palabras clave: relave, corte simple cíclico, respuesta drenada, CSR
Classified coarse grained tailings, also called sand tailings, are used to construct the main embankment wall in tailings dams. Due to the importance of this structure in the global stability of the dam, the wall is designed to resist different type of loading, e.g. monotonic loading, seepage from the pond, dynamic loading, wind erosion, among others. Within dynamic loading, special concern is usually given to the undrained response of the material since it may trigger liquefaction phenomena (e.g. cyclic mobility type of response). On the other hand, the cyclic drained response has usually received little attention despite its potential implications. This is likely due to the series of international standards that specify a minimum operational and total freeboard in the scenario of excessive crest settlements. However, is uncertain if such requirements are adequate for a series of scenarios related to the in-situ characteristics of the wall and earthquake loading. In this context, the present study shows results of a series of drained Direct Simple Shear (DSS) tests conducted on sand tailings prepared under different conditions. Results are presented in terms of settlement versus applied cyclic stress. In addition, monotonic tests were conducted in order to preliminary explore a relationship between the critical state line (through the critical state parameter) and the observed cyclic response.

Keywords: tailings, direct simple shear, drained response, CSR 


\section{Introducción}

Existen una serie de fenómenos físicos que, combinados con una deficiente construcción, pueden provocar el colapso de depósitos de relaves, por ejemplo, fallas por overtopping, licuefacción, falla de taludes, extrusión lateral, etc. En países altamente sísmicos como Chile, un gran número de fallas se atribuyen a la ocurrencia de licuefacción inducida sísmicamente (falla de flujo o movilidad cíclica). Esto se observó en 1965 después de la ruptura del tranque El Cobre Viejo y más recientemente en el Terremoto de Maule 2010, donde se visualizaron fallas locales en depósitos (Villavicencio et al., 2014). Es por esto que las regulaciones locales (Decreto Supremo $\left.\mathrm{N}^{\circ} 248,2007\right)$, así como la práctica de la ingeniería, tienden a enfocar sus requerimientos y esfuerzos en el estudio de la respuesta sísmica saturada no drenada de los muros de tranques de relaves. Por otra parte, la respuesta cíclica drenada de las arenas de relaves no suele ser considerada en análisis experimentales en etapas de diseño u operación, debido a cargas sísmicas o de otro origen (vibración de maquinaria).

Las arenas de relaves en diversos estados de saturación se suelen asentar frente a cargas cíclicas en condiciones drenadas, causando el asentamiento del muro y reduciendo por tanto la revancha. La provisión de una revancha es importante debido a que genera un margen para evitar el desbordamiento del material almacenado, incluyendo los eventuales almacenamientos por fuertes lluvias o problemas operacionales. Por otro lado, una revancha insuficiente puede aumentar la susceptibilidad del tranque a experimentar erosión hidráulica y rebalse. Las regulaciones locales e internacionales establecen un mínimo de revancha requerido para satisfacer una serie de escenarios adversos; por ejemplo, la guía de seguridad de presas de Finlandia requiere que la revancha exceda la altura máxima de ola o la profundidad de penetración según el índice de congelación (Witt et al., 2004). En Sudáfrica, la revancha debe ser mayor en $0.8 \mathrm{~m}$ respecto a la tormenta más desfavorable por 24 horas con un periodo de retorno de 50 años (Water \& Forestry, 2008). En Chile, en cambio, se requiere una revancha mínima de $1 \mathrm{~m}$ pudiendo ser superior en casos que los fenómenos climatológicos lo demanden (Decreto Supremo N²48, 2007). Pese a estas recomendaciones generales, existen aún una serie de incertidumbres respecto a la respuesta drenada de arenas de relaves ante una serie de consideraciones de estado del material (relación de vacíos, presión de vertical, humedad) y demandas sísmicas.

En este contexto, el presente estudio tiene como objetivo explorar la respuesta cíclica drenada de arena de relaves considerando la teoría de estado crítico. Para lograr lo anterior, se utilizó un aparato de corte simple cíclico (Direct Simple Shear, DSS) que permitió ejecutar ensayos drenados en una arena de relaves a distintas presiones de consolidación y densidades relativas. Por último, este estudio propone una serie de correlaciones simplificadas que permiten cuantificar el asentamiento de muros bajo cargas cíclicas y en consideración del parámetro de estado inicial.

\section{Metodología \\ Materiales}

La arena utilizada en el estudio corresponde a un relave de cobre que clasifica según el Sistema de Clasificación de Suelos Unificado (USCS) como una arena limosa (SM). Posee un índice de plasticidad de 0, una gravedad específica de 2.83 y un peso máximo y mínimo unitario seco de 18 y $14 \mathrm{kN} / \mathrm{m}^{3}$, respectivamente.

\section{Aparato de ensayo}

Las pruebas cíclicas se realizaron usando un dispositivo de corte simple (Direct Simple Shear, DSS) de volumen constante. Este aparato es una variación del tipo desarrollado en el Norwegian Geotechnical Institute (NGI) en la década de 1960. El DSS simula la rotación cíclica de los esfuerzos principales que se producen durante un terremoto (Wijewickreme et al., 2005), recreando ciclos equivalentes y permitiendo la consolidación de las muestras en condiciones de $K_{0}$. En una condición drenada, se libera el movimiento vertical de la muestra, permitiendo su compresión o expansión (dilatancia) (Suazo et al., 2016). Las dimensiones de las muestras son de $70 \mathrm{~mm}$ en diámetro y altura inicial aproximada de $22 \mathrm{~mm}$. En este estudio, el esfuerzo cíclico se aplicó a una frecuencia de $0.2 \mathrm{~Hz}$. Las muestras se prepararon a densidades relativas (DR) de $65 \%$ y $80 \%$ con el contenido de agua óptimo según la prueba Proctor. Las DR utilizadas corresponden al $92 \%$ y $95 \%$ de la densidad seca máxima de la prueba 
Proctor normalizada. Se consideraron valores de razón de esfuerzo de corte cíclico CSR, definido como $\tau_{c y} / \sigma_{v}^{\prime}$, donde $\tau_{c y}$ es el esfuerzo cíclico de corte $\mathrm{y} \sigma_{v}^{\prime}$ es la presión de consolidación efectiva, que van desde 0.1 a 0.4 . Las pruebas fueron detenidas cuando se observaron cambios insignificantes de altura en la muestra (siempre inferior a los 300 ciclos).

\section{Resultados y análisis}

\section{Línea de compresión normal y línea de estado crítico}

Se realizaron una serie de pruebas monótonas, corte simple y edómetro, en condiciones drenadas a diferentes densidades relativas y presiones verticales para definir la línea de estado crítico CSL y la línea de compresión normal NCL para el material utilizado. Los resultados se presentan en la Figura 1. Se observa que las curvas de DR $=65 \%$ y $80 \%$ se encuentran en estado suelto y denso respecto a la CSL, respectivamente, lo que facilita la interpretación de los resultados presentados en el estudio. Para caracterizar la respuesta, se utilizó el parámetro de estado crítico $\psi$ propuesto por Been y Jefferies (1985), donde $e_{0}$ es la relación de vacíos respecto a la CSL dado por (1).

$$
\psi=e_{0}-e_{\mathrm{CSL}}
$$

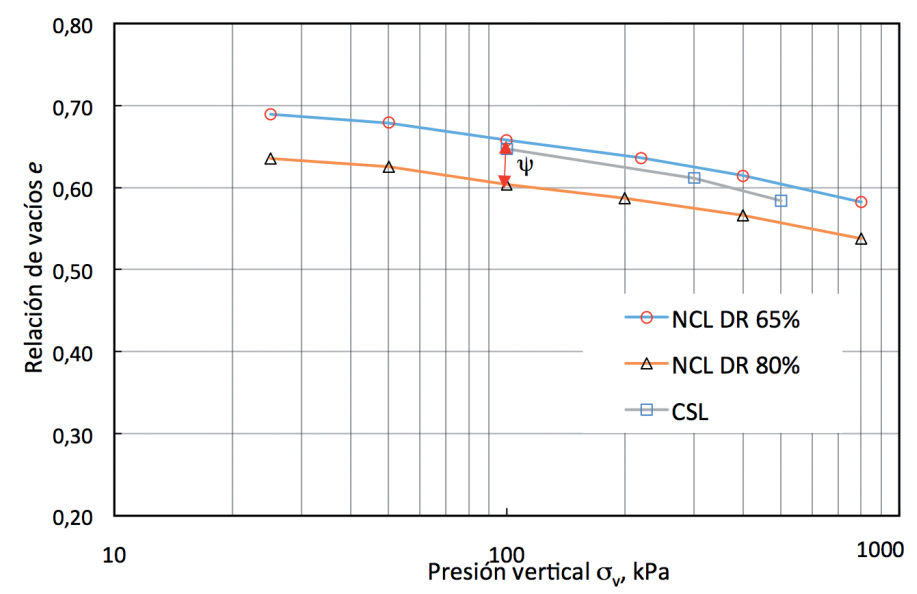

Figura 1: Línea de Estado Crítico (CSL) y Línea de Compresión Normal (NCL) para el material utilizado

\section{Respuesta drenada versus CSR}

Se realizaron un total de 12 pruebas de DSS a valores de CSR que oscilan entre 0.1 y 0.4. La Figura 2 muestra el comportamiento drenado en términos de cambio de relación de vacíos versus número de ciclos para muestras preparadas a densidades relativas de 65 y $80 \%$, y consolidadas a $100 \mathrm{kPa}$. Se puede observar que el material solo experimenta asentamientos si el CSR aplicado es mayor que la CSR máximo experimentado previamente (en el pasado). Lo anterior es válido, siempre y cuando se alcance el equilibrio (no existe cambio de volumen significativo entre ciclos consecutivos) de deformación al CSR seleccionado. Se observa también que a medida que disminuye la relación de vacíos, se requiere un valor mayor de CSR para causar asentamientos. Lo anterior es significativo, dado que, para una relación de vacíos dada, existe un valor umbral de CSR sobre el cual se producen cambios de volumen bajo cargas cíclicas. Bajo este valor, el relave no se asentaría, independiente del número de ciclos impuesto. Esto se discute en más detalle en la siguiente sección.

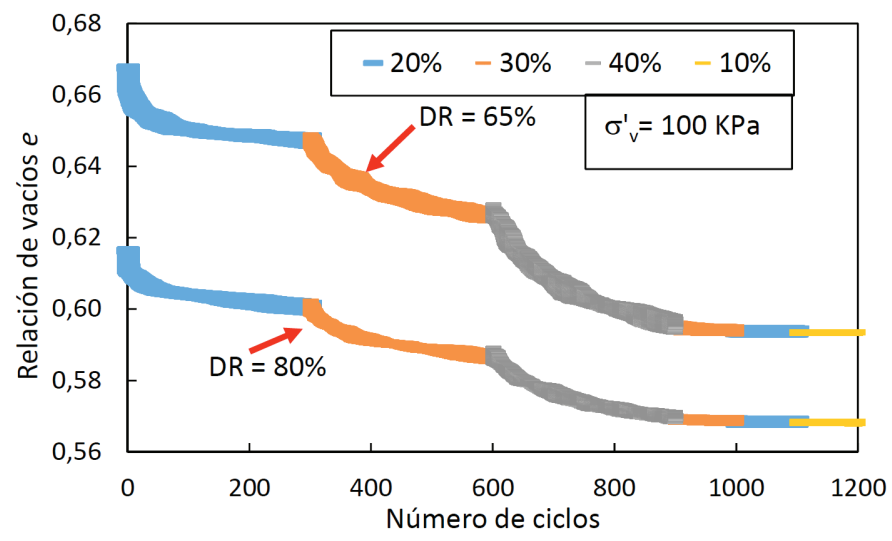

Figura 2: Cambio de la relación de vacíos frente a la aplicación de sucesivos CSR (0.1 a 0.4) para muestras preparadas a una DR $=65 \%$ y $80 \%$

\section{Evolución del parámetro de estado crítico}

La Figura 3a muestra la evolución del parámetro de estado crítico. Los resultados se presentan en términos del parámetro de estado inicial $\psi_{0} \mathrm{y}$ de aquel resultante luego de la aplicación de la carga cíclica $\psi_{f}$. En la figura, se han definido tres estados posibles para los relaves de arena: i) Estado I, donde el suelo permanece suelto, es decir, por sobre la CSL después de la carga aplicada (debido a las bajas tensiones cíclicas); ii) Estado II , donde el suelo inicialmente se encuentra suelto respecto a la CSL, pero después de la carga cíclica pasa a un estado denso, bajo la CSL, debido al comportamiento contractivo experimentado; y iii) Estado III, donde los parámetros iniciales y finales están por debajo de la CSL, caso denso 
(en este caso se requiere un mayor CSR para provocar un cambio). Los diferentes estados están limitados por una línea definida por los autores como Línea de no Asentamiento. Si el suelo que se encuentra en esta línea, no experimentará cambios de volúmenes, a pesar de la aplicación de cargas cíclicas. La Figura $3 \mathrm{~b}$ muestra la misma gráfica, pero considerando las cargas extremas, es decir, CSR de 0.1 y 0.4. Se puede observar que para la menor solicitación se producen pequeños cambios en $\psi$, por lo tanto, el suelo permanece cercano a la línea de no asentamiento o en el Estado I. Por el contrario, para el CSR de 0.4; hay una transición inmediata del estado suelto al estado denso (Estado II). Los suelos densos se caracterizan por comenzar con un parámetro de estado negativo, y para CSR pequeños $(0.1$ y 0.2$)$ estarán sobre la línea de no asentamiento o cercana a ella, mientras que grandes CSR (0.3 y 0.4) tendrán un cambio, estando en el Estado III.

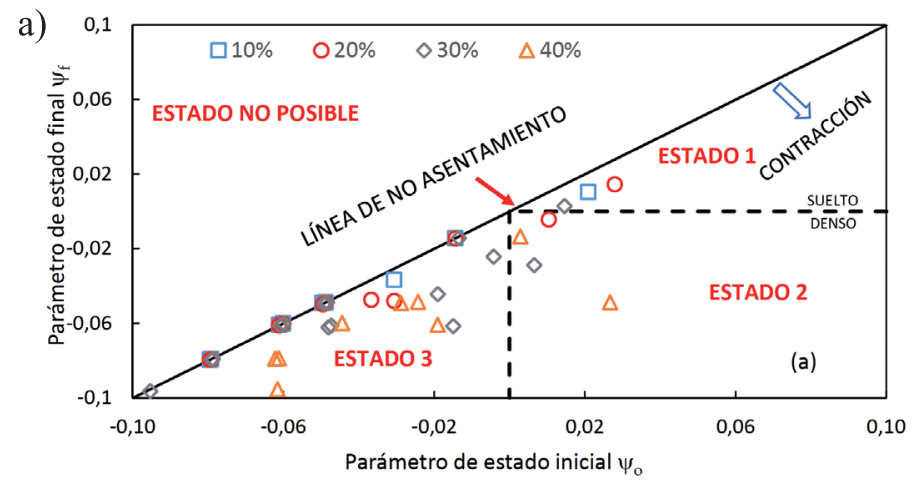

b)

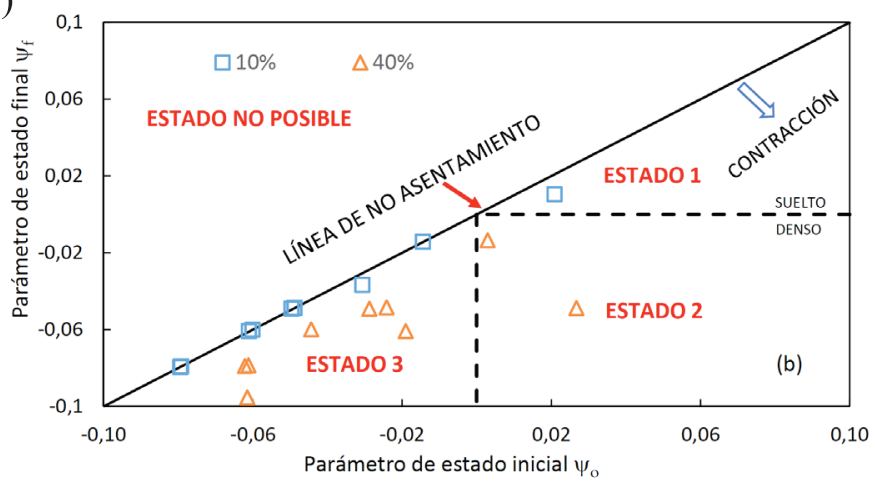

Figura 3: Evolución del parámetro de estado crítico para cagas cíclicas. a) Todos los datos y b) respuesta para CSR 0.1 y 0.4

\section{Implicancia práctica}

Si se selecciona un CSR para un número equivalente de 15 ciclos, i.e. relación de resistencia cíclica, CRR, es posible explorar los asentamientos para eventos sísmicos de magnitud $M=7.5$ (Idriss y Boulanger, 2010). Esto se presenta en la Tabla 1, incluyendo además el cambio de altura para 300 ciclos (establecidos como suficientes para alcanzar equilibrio de deformación para cada carga cíclica aplicada). De la tabla, se puede observar que bajo el escenario de un sismo que sea capaz de generar grandes aceleraciones, el tranque experimentaría asentamientos aproximados del $2 \%$ de su altura para una densidad relativa del $65 \%$. Sin embargo, si se encuentra compactado de manera adecuada, la deformación se reduce a un $1.7 \%$, aproximadamente. Es decir, si el tranque posee $100 \mathrm{~m}$ de altura, este podría asentarse como máximo $1.7 \mathrm{~m}$, lo que parece coherente con las recomendaciones generales de revancha empleadas.

En la Figura 4, se presentan los resultados de la Tabla 1 en términos de carga cíclica aplicada y la razón de asentamiento. Es posible observar un buen ajuste de la data experimental con un aumento significativo de la razón de asentamiento con el valor de CSR. Es preciso señalar, que los datos experimentales presentados aquí, provienen de muestras consolidadas a $100 \mathrm{kPa}$. En la siguiente sección se presenta como afecta el cambio de la presión de consolidación en el asentamiento en total.

Tabla 1: Asentamiento esperado para un CSR dado

\begin{tabular}{|c|c|c|c|c|c|}
\hline \multirow{2}{*}{$\begin{array}{c}\text { DR, } \\
\%\end{array}$} & \multirow{2}{*}{ CSR } & \multicolumn{2}{|c|}{ Máximo registrado } & \multicolumn{2}{c|}{ Terremoto 7.5} \\
\cline { 2 - 6 } & & Ciclos & $\Delta H / H, \%$ & Ciclos & $\Delta H / H, \%$ \\
\hline \multirow{4}{*}{65} & 0.1 & 300 & 0.63 & 15 & 0.39 \\
\cline { 2 - 6 } & 0.2 & 300 & 1.30 & 15 & 0.74 \\
\cline { 2 - 6 } & 0.3 & 300 & 2.14 & 15 & 1.29 \\
\cline { 2 - 6 } & 0.4 & 300 & 4.51 & 15 & 2.13 \\
\hline \multirow{4}{*}{80} & 0.1 & 300 & 0.37 & 15 & 0.20 \\
\cline { 2 - 6 } & 0.2 & 300 & 1.08 & 15 & 0.54 \\
\cline { 2 - 6 } & 0.3 & 300 & 1.56 & 15 & 0.94 \\
\cline { 2 - 6 } & 0.4 & 300 & 2.57 & 15 & 1.76 \\
\hline
\end{tabular}

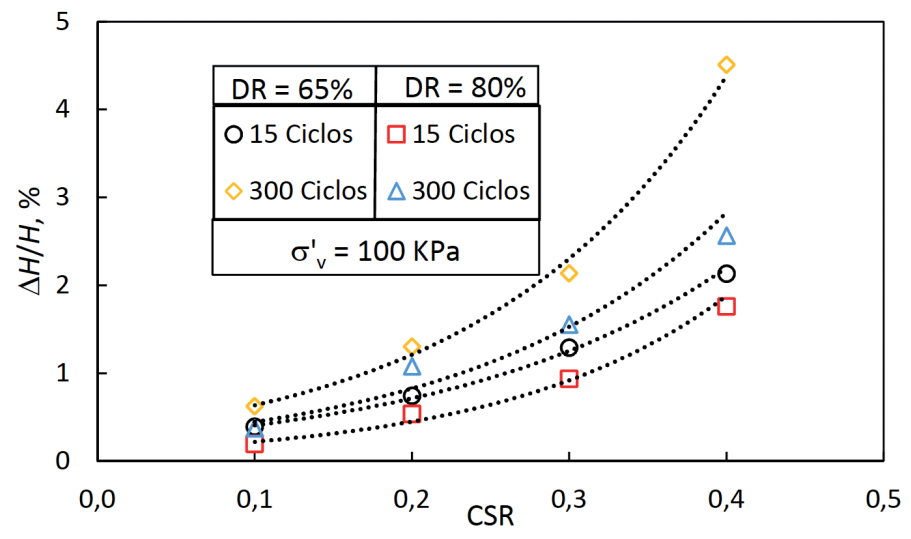

Figura 4: Asentamiento esperado para un CSR dado 


\section{Efecto del esfuerzo de consolidación}

En la Figura 5 se presenta la razón de asentamiento para cuatro presiones verticales efectivas pero sometidas al mismo CSR de 0.3. En esta figura, las muestras fueron preparadas a una $\mathrm{DR}=65 \%$, y la carga cíclica fue hasta los 150 ciclos equivalentes. Se observa un aumento del asentamiento a mayores presiones de consolidación. Comparando con el análisis no drenado, es consistente con el efecto de la presión de confinamiento en el valor de CRR $\left(K_{\mathrm{o}}\right)$ para arenas con bajos contenidos de finos, debido a que a mayor esfuerzo de consolidación menor es el factor de corrección $K_{\sigma}$, disminuyendo la resistencia, según discuten Suazo et al. (2016). Sin embargo, aún falta mayor estudio y resultados de ensayos para poder comprender el comportamiento en el caso drenado.

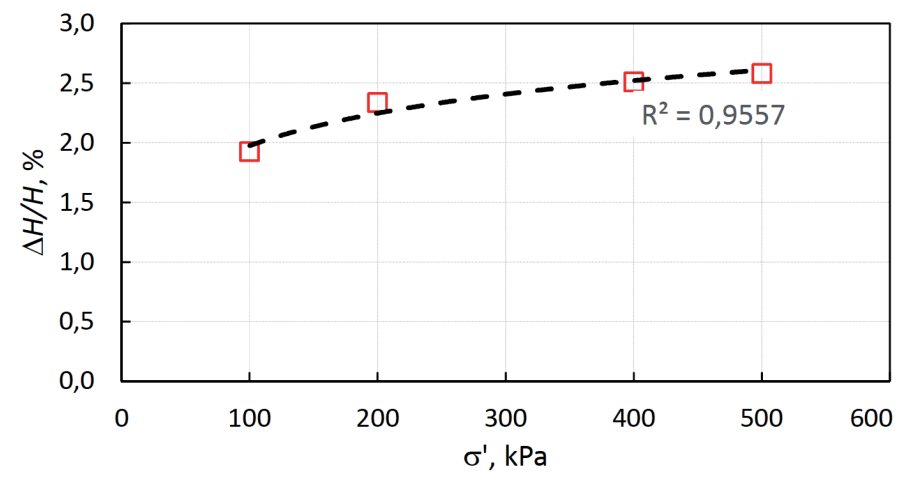

Figura 5: Cambio del asentamiento frente a distintos esfuerzos de consolidación, $\mathrm{DR}=65 \%$

\section{Conclusiones}

Este artículo exploró la respuesta drenada de arenas de relaves. Se observó que los asentamientos ocurren sólo si la carga sísmica actual es superior a cualquier evento sísmico anterior (suponiendo un gran número de ciclos equivalentes). También se propuso que existe una fuerte correlación entre el asentamiento, CSR y el parámetro de estado crítico. Los resultados de los asentamientos obtenidos, parecen consistentes con la revancha considerada en la práctica y normativa internacional. Sin embargo, estos resultados son pocos conservadores, debido a que el sismo evaluado dependiendo de la zona sísmica puede ser mucho mayor, se necesitan mayor investigación para poder generar expresiones generales para distintos tipos de relaves y evaluar condiciones de expansión lateral. Por tanto, es recomendable en cualquier diseño de depósitos, considerar pruebas especiales de respuesta drenada.

\section{Agradecimientos}

Los autores agradecen el apoyo brindado por el personal del Laboratorio de Geotecnia de la Universidad Técnica Federico Santa María (LEMCO). También quisiéramos reconocer el apoyo financiero proporcionado por PMI Ines FSM1402_B21 dirigido por el Profesor Gonzalo Suazo y a la Dirección General de Investigación, Innovación y Postgrado (DGIIP).

\section{Referencias}

Been, K. and Jefferies, M.G. (1985). A state parameter for sands. Géotechnique 35(2), 99-112

Decreto Supremo N²48 (2007). Reglamento para la aprobación de proyectos de diseño, construcción, operación y cierre de los depósitos de relaves. Ministerio de Minería, Chile

Idriss I.M. and Boulanger, R.W. (2010). SPT-based liquefaction triggering procedures. Report No. UCD/CGM-10-02, Center for Geotechnical Modeling, University of California Davis, USA

Suazo, G., Fourie, A., Doherty, J. and Hasan, A. (2016). Effects of confining stress, density and initial static shear stress on the cyclic shear response of fine-grained unclassified tailings. Géotechnique 66(5), 401-412

Villavicencio, G., Espinace, R., Palma, J., Fourie, A. and Valenzuela, P. (2014). Failures of sand tailings dams in a highly seismic country. Canadian Geotechnical Journal 51(4), 449-464

Water \& Forestry (2008). Water management for mine residue deposits. Department of Water Affairs and Forestry. Republic of South Africa.

Wijewickreme, D., Sanin, M. and Greenaway, R. (2005). Cyclic shear response of fine-grained mine tailings. Canadian Geotechnical Journal 43(5), 1408-1421

Witt, K.J., Schönhardt, M., Saarela, J. and Frilander, R. (2004). Sustainable improvement in safety of tailings facilities. Report on Tailings Management Facilities-Risks and Reliability, TAILSAFE: A European Research and Technological Development Project, http://www. tailsafe. com 